\title{
Spectral shadowing suppression technique in phase-OTDR sensing based on weak fiber Bragg grating array
}

\author{
Veronica de Miguel Soto, ${ }^{1, *}$ (1) Johan Jason, ${ }^{2}$ Deniz Kurtoğlu, ${ }^{2,3}$ Manuel Lopez-Amo, ${ }^{1}$ and \\ MARC WUILPART $^{2}$ \\ ${ }^{1}$ Department of Electrical and Electronic Engineering, Public University of Navarra and ISC, Campus de Arrosadia, 31006 Pamplona, Spain \\ ${ }^{2}$ Department of Electromagnetism \& Telecommunications, Faculty of Engineering, University of Mons, 7000 Mons, Belgium \\ ${ }^{3}$ Department of Electrical and Electronics Engineering, Izmir Institute of Technology, Urla, 35430 Izmir, Turkey \\ *Corresponding author: veronica.demigue/@unavarra.es
}

Received 26 September 2018; revised 14 December 2018; accepted 2 January 2019; posted 2 January 2019 (Doc. ID 346808); published 18 January 2019

\begin{abstract}
A postprocessing procedure is presented to suppress spectral shadowing in phase-OTDR sensing systems based on a weak fiber Bragg grating array. A complete theoretical analysis of the interfering signals has been carried out to identify a compensation method. The proposed approach has been applied to simulated and experimental phase-OTDR in the context of vibration measurements. Fast Fourier transform has been employed to analyze the obtained results, which has verified the validity of the proposed method to suppress spectral shadowing. (๑) 2019 Optical Society of America
\end{abstract}

https://doi.org/10.1364/OL.44.000526

Provided under the terms of the OSA Open Access Publishing Agreement

Structural health monitoring (SHM) has attracted more attention in both scientific and engineering fields in recent years $[1,2]$. The ability of detecting internal failures of a structure and providing early warnings of structural damages or decays becomes crucial. SHM can be implemented in all kinds of civil structures, e.g., dams, tunnels, highways, railways, bridges, pipelines. [3]. The main parameters to consider when interrogating the health condition of structures are load, deformation, strain, temperature, and vibration.

Optical fiber sensors present specific advantages to be exploited in SHM systems, such as lightweight and geometric versatility. Among them, fiber Bragg gratings (FBGs) offer a huge capacity for mechanical sensing applications [4]. In addition, FBGs allow multiplexing and then multipoint sensing schemes, placing many gratings within a single optical fiber. However, the spectral ranges of the emitting and detecting devices limit the number of sensing points. One way to solve this limitation is by using distributed optical fiber sensing systems. These systems present a large-scale monitoring range, large numbers of monitored points, simple deployment, and geometric versatility compared with point sensors [5].

Among the existing distributed systems, phase-sensitive optical time domain reflectometry $(\Phi-\mathrm{OTDR})$ has proven to be a power- ful tool for real-time distributed sensing. Various events can be monitored, such as borderline intrusion, seismic waves, and train movement $[6,7]$. In $\Phi$-OTDR systems, a light source with a narrow linewidth and minimum frequency shift is employed. Light is pulsed and later injected into a conventional single-mode fiber. The multiple scattering centers within the resolution cell, i.e., a zone equal to half the pulse width, generates backscattered light components that interfere coherently at the $\Phi$-OTDR detector. By measuring the variation of the coherent superposition of the backscattered light, any perturbation can be identified and localized along the sensing fiber.

The signal-to-noise ratio (SNR) of $\Phi$-OTDR is limited by the weak Rayleigh backscattering generated in the fiber. Using an FBG array is a potential method to improve the SNR in certain zones of a Rayleigh-based OTDR system, as presented in Ref. [8]. For the phase-OTDR application, the FBGs act as individual scattering centers with well-defined positions and reflectivities. Therefore, the phase-OTDR is required to operate in the regime of registering FBG reflections, rather than Rayleigh backscattered light from the fiber. By interrogating an array of FBG pairs, it is possible to detect any phase variation appearing between two FBGs of one pair, as long as they are separated by a distance smaller than the resolution cell $[9,10]$. Another condition is to separate each consecutive pair by a distance larger than the resolution cell to prevent signals from more than two FBGs to interfere simultaneously [9].

However, the spectral shadowing crosstalk effect [11] is a limiting factor in these systems. The interrogating light needs to pass all upstream FBGs to reach a specific FBG. As a result, the light illuminating a certain FBG in the array carries the spectral features of all the previous ones. Accordingly, it shadows the response of the actual FBG. The resulting error has been studied in Ref. [11] in the context of a quasidistributed fiber sensor interrogated by an OFDR (optical frequency domain reflectometer). The parasitic effects present in the scheme have been simulated and enhancement treatments proposed. 
In this letter, the spectral shadowing effect in a $\Phi$-OTDR system based on an array of FBG pairs is analyzed. A detailed study of the signals involved in the scheme has allowed the development of a postprocessing method which suppresses the spectral shadowing effect. The performance of the compensation technique is demonstrated by simulations and then experimentally examined for vibration measurements. Finally, an estimation of the maximum number of FBG pairs possible to interrogate with the proposed technique is provided.

The experimental setup used in this work is presented in Fig. 1. It is formed by three different parts: the source, the receiver, and the fiber under test (FUT). First, an ultra-narrow linewidth laser (NLL) emitting highly coherent, continuous light is employed as the light source, having a linewidth of $0.1 \mathrm{kHz}$ and a wavelength of $1552.5 \mathrm{~nm}$. Then, an acousto-optic modulator (AOM) with a $160 \mathrm{MHz}$ frequency shift creates probe pulses with $100 \mathrm{~ns}$ width at a $20 \mathrm{kHz}$ repetition rate. The probe pulses are amplified by an erbium doped fiber amplifier (EDFA), then filtered by a $0.9 \mathrm{~nm}$ band pass filter and finally injected into the FUT through port one of a three-port optical circulator. The backscattered/reflected light is directed to the receiver through port three for detection. The receiver consists of a photo detector (PD) with a transimpedance gain amplifier and a data acquisition card (DAQ) with $1 \mathrm{GS} / \mathrm{s}$ sampling rate. Finally, the variation in detected power over time is registered and analyzed.

Spectral shadowing is relevant when a light source illuminates a concatenation of FBGs with overlapping spectral features. As a result, distortion builds up along the fiber. To study the effects of spectral-shadowing crosstalk, two pairs of ultra-low reflectivity $(0.02 \%)$ FBGs have been employed. The four FBGs inscribed in the FUT essentially share the same characteristics, having a center wavelength of $1552.5 \mathrm{~nm}$, a $3 \mathrm{~dB}$ bandwidth of $0.2 \mathrm{~nm}$, and a length of $4 \mathrm{~mm}$. The grating pitch is $536.42 \mathrm{~nm}$, and the average refractive index modulation is $1.6 \times 10^{-5}$ with an effective refractive index of 1.4471 . A lead-in fiber spool of $1.5 \mathrm{~km}$ is followed by the set of FBGs and a terminating fiber spool of $1 \mathrm{~km}$ length. The two pairs are separated by approximately $30 \mathrm{~m}$, and the two FBGs in each pair are separated by $4 \mathrm{~m}$. In the present study, the large distance applied between the FBG pairs is for visibility reasons. However, the formalism and suppression technique developed can directly be applied to equidistant FBG arrays. The $4 \mathrm{~m}$ fiber length between the first two FBGs $\left(\mathrm{FBG}_{1}\right.$ and $\left.\mathrm{FBG}_{2}\right)$ and $\mathrm{FBG}_{2}$ itself are attached to a plastic tube connected at its midpoint to a shaker (SHR1), leaving $\mathrm{FBG}_{1}$ static.

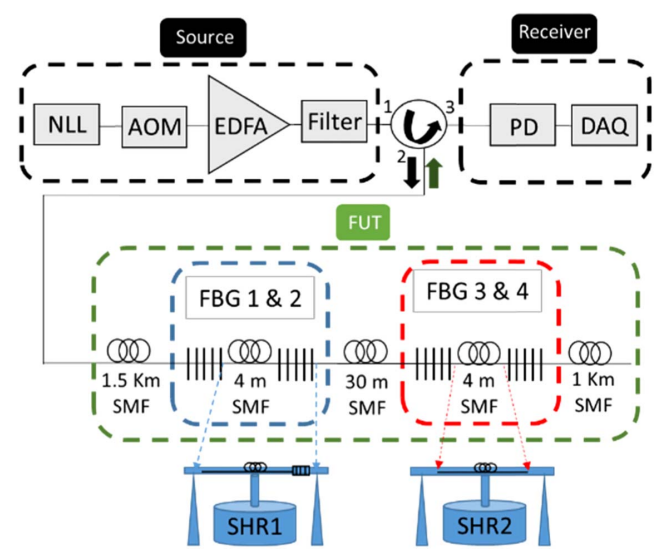

Fig. 1. Experimental setup.
Additionally, the $4 \mathrm{~m}$ section between $\mathrm{FBG}_{3}$ and $\mathrm{FBG}_{4}$ is attached to another plastic tube connected at its midpoint to shaker SHR2, while $\mathrm{FBG}_{3}$ and $\mathrm{FBG}_{4}$ are static. Both plastic tubes have their ends clamped. In the experiment, SHR2 is driven by a sinusoidal signal at $2 \mathrm{kHz}$ with $0.1 \mathrm{~g}$ acceleration amplitude, and SHR1 is driven by two superposed sinusoidal signals at 300 and $700 \mathrm{~Hz}$, each with a $1 \mathrm{~g}$ acceleration amplitude.

The scheme of the signals involved for a single grating pair in an array is presented in Fig. 2. If the rectangular pulse injected into the FUT has a width $W$ greater than twice the separation $L$ between $\mathrm{FBG}_{A}$ and $\mathrm{FBG}_{B}$, the signals reflected from both FBGs overlap in a zone of length $W / 2-L$ of the $\phi$-OTDR trace. Due to the narrow linewidth of the laser, the coherence is maintained when the reflected signals overlap at the detector and interference occurs. The optical power of the reflected signal from $\mathrm{FBG}_{A}$ $\left(\mathrm{FBG}_{B}\right)$ detected by the $\phi$-OTDR is denoted by $P_{A}\left(P_{B}\right)$ and the optical power of the interference signal by $P_{A B}$.

The theoretical analysis of the signals detected in the system is presented below. The particular features of each FBG (effective refractive index, index modulation depth, periodicity, and grating length) determine the magnitude and phase of the reflected and the transmitted signals through the complex reflection and transmission coefficients $\mathbf{r}$ and $\boldsymbol{t}$, as provided in Ref. [12]. In the experiment, the $\phi$-OTDR launches $100 \mathrm{~ns}$ pulses (resulting in a resolution cell $W / 2=10 \mathrm{~m}$ ). Given a complex electric field, $\boldsymbol{E}_{\mathrm{in}}$, at the FUT input, a complex reflection coefficient for $\mathrm{FBG}_{A}\left(\mathrm{FBG}_{B}\right) \boldsymbol{r}_{A}\left(\boldsymbol{r}_{\boldsymbol{B}}\right)$ and a complex transmission coefficient, $\boldsymbol{t}_{\boldsymbol{A}}$, for $\mathrm{FBG}_{A}$, the electric fields $\boldsymbol{E}_{\boldsymbol{A}}$ and $\boldsymbol{E}_{\boldsymbol{B}}$ reflected from $\mathrm{FBG}_{A}$ and $\mathrm{FBG}_{B}$, respectively, are:

$$
\begin{gathered}
\boldsymbol{E}_{\boldsymbol{A}}=\boldsymbol{E}_{\mathrm{in}} \boldsymbol{T}^{2}(t) \boldsymbol{r}_{\boldsymbol{A}}(t), \\
\boldsymbol{E}_{\boldsymbol{B}}=\boldsymbol{E}_{\mathrm{in}} \boldsymbol{T}^{2}(t) \boldsymbol{t}_{\boldsymbol{A}}^{2}(t) \boldsymbol{r}_{\boldsymbol{B}}(t) e^{j \Delta \varphi(t),}
\end{gathered}
$$

where $\boldsymbol{T}(t)$ is the product of the complex transmission coefficients of all FBGs preceding $\mathrm{FBG}_{A}$, and $\Delta \varphi(t)$ is twice the phase difference induced between $\mathrm{FBG}_{A}$ and $\mathrm{FBG}_{B}$. $\Delta \varphi(t)$ contains information about the perturbation (vibration) applied between $\mathrm{FBG}_{A}$ and $\mathrm{FBG}_{B}$. Using Eqs. (1) and (2), the interference signal, $\boldsymbol{E}_{A B}$, at the detector is given by

$$
\begin{aligned}
\boldsymbol{E}_{A B} & =\boldsymbol{E}_{\boldsymbol{A}}+\boldsymbol{E}_{\boldsymbol{B}} \\
& =\boldsymbol{E}_{\mathrm{in}} \boldsymbol{T}^{2}(t) \boldsymbol{r}_{\boldsymbol{A}}(t)+\boldsymbol{E}_{\mathrm{in}} \boldsymbol{T}^{2}(t) \boldsymbol{t}_{A}^{2}(t) \boldsymbol{r}_{\boldsymbol{B}}(t) e^{j \Delta \varphi(t)} .
\end{aligned}
$$

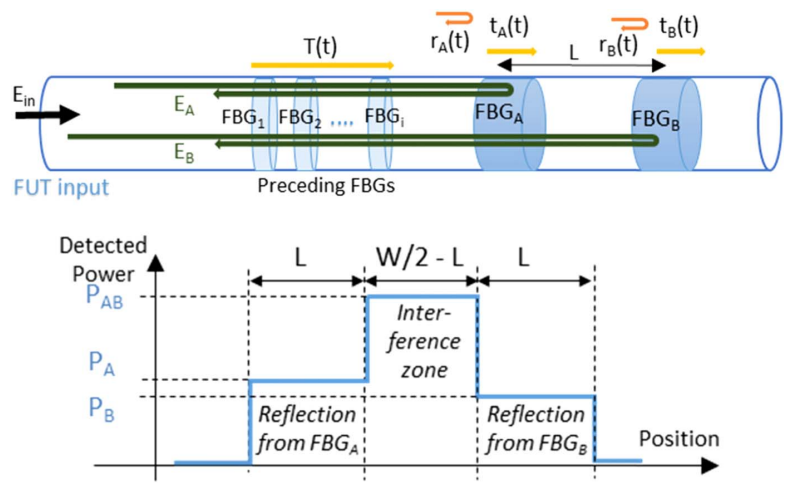

Fig. 2. Scheme of the theoretical analysis of reflected signals from a single FBG pair, including power levels of the corresponding $\phi$-OTDR signature. 
The power reflected from the FBG pair and detected by the phase-OTDR presents three different zones (Fig. 2) with corresponding powers $P_{A}, P_{B}$, and $P_{A B}$ :

$$
\begin{gathered}
P_{A}=\boldsymbol{E}_{A} \boldsymbol{E}_{\boldsymbol{A}}^{*}=\left|\boldsymbol{E}_{\mathrm{in}}\right|^{2}|\boldsymbol{T}(t)|^{4}\left|\boldsymbol{r}_{\boldsymbol{A}}(t)\right|^{2}, \\
P_{B}=\boldsymbol{E}_{\boldsymbol{B}} \boldsymbol{E}_{\boldsymbol{B}}^{*}=\left|\boldsymbol{E}_{\mathrm{in}}\right|^{2}|\boldsymbol{T}(t)|^{4}\left|\boldsymbol{t}_{\boldsymbol{A}}(t)\right|^{4}\left|\boldsymbol{r}_{\boldsymbol{B}}(t)\right|^{2} \\
P_{A B}=\boldsymbol{E}_{A B} \boldsymbol{E}_{A B}^{*}=\left|\boldsymbol{E}_{\mathrm{in}}\right|^{2}|\boldsymbol{T}(t)|^{4}\left|\boldsymbol{r}_{A}(t)\right|^{2} \\
+\left|\boldsymbol{E}_{\mathrm{in}}\right|^{2}|\boldsymbol{T}(t)|^{4}\left|\boldsymbol{t}_{\boldsymbol{A}}(t)\right|^{4}\left|\boldsymbol{r}_{\boldsymbol{B}}(t)\right|^{2} \\
+2\left|\boldsymbol{E}_{\mathrm{in}}\right|^{2}|\boldsymbol{T}(t)|^{4}\left|\boldsymbol{t}_{\boldsymbol{A}}(t)\right|^{2}\left|\boldsymbol{r}_{\boldsymbol{A}}(t)\right|\left|\boldsymbol{r}_{\boldsymbol{B}}(t)\right| \cos (\Delta \varphi(t)+\theta(t)),
\end{gathered}
$$

where $\theta(t)=\arg \left[r_{B}(t) / r_{A}(t)\right]$. Because $\boldsymbol{T}(t)$ is the product of the transmission coefficients of all preceding FBGs, it depends on the spectral properties of preceding gratings and influences the power detected for the interference signal, $P_{A B}$. Using Eqs. (4)-(6), the following expression can be calculated:

$$
\cos (\Delta \varphi(t)+\theta(t))=\frac{P_{A B}(t)-P_{A}(t)-P_{B}(t)}{2\left[\left(P_{A}(t)\right)^{1 / 2}\left(P_{B}(t)\right)^{1 / 2}\right]} .
$$

By reading out the above three power values from the $\phi$-OTDR trace, the change in the phase component $\Delta \varphi(t)+\theta(t)$ occurring upon any events between $\mathrm{FBG}_{A}$ and $\mathrm{FBG}_{B}$ can be monitored. As a result, the undesired spectral shadowing from preceding FBGs is eliminated $(|\boldsymbol{T}(t)|$ is suppressed). It is noted that the polarization mismatch between the lightwaves reflected by $\mathrm{FBG}_{A}$ and $\mathrm{FBG}_{B}$ is not expressed in Eq. (6). The mismatch can be considered by multiplying the interference term of $P_{A B}$ by a factor $k$ ranging between 0 and 1 [10]. However, this effect does not change the compensation ability of our approach, because $|\boldsymbol{T}(t)|$ is still suppressed. $k$ should be nonzero, as assumed in Refs. $[9,10]$. This can be ensured by a proper choice of the fiber birefringence properties [10].

Simulations and experiments have been performed based on the setup depicted in Fig. 1 to validate the spectral shadowing compensation technique. Simulations were performed in Matlab by computing $3000 \phi$-OTDR traces with a time separation of $50 \mu \mathrm{s}(20 \mathrm{kHz}$ pulse repetition rate) and a sampling resolution of $0.1 \mathrm{~m}$ (1 GS/s DAQ sampling rate). Each trace was computed as a function of the pulse position using Eqs. (4)-(6), considering a rectangular pulse shape and neglecting the Rayleigh backscattering signal and the fiber attenuation. The vibration induced by the shakers were simulated by introducing variations $\Delta n=\Delta n_{m} \sin (2 \pi f t)$ in the effective refractive index in each part of the FUT being subject to vibration. As in the experimental setup, the frequency of vibration $f$ was set to $300 \mathrm{~Hz} / 700 \mathrm{~Hz}(2 \mathrm{kHz})$ for SHR1 (SHR2), assuming a maximum refractive index change $\Delta n_{m}$ of $10^{-5}\left(10^{-8}\right)$ induced by the vibration.

Figure 3(a) shows the superposed $\phi$-OTDR traces obtained from a simulation with the given parameters. The interference zones of the two FBG pairs are identified by $S_{12}=18-24 \mathrm{~m}$ and $S_{34}=52-58 \mathrm{~m}$ showing the largest change in detected power over time. To determine the frequency of the induced vibration, FFT analysis was performed at a position within the $S_{34}$ zone. Likewise, the frequency of vibration at other points of the FUT can be determined.

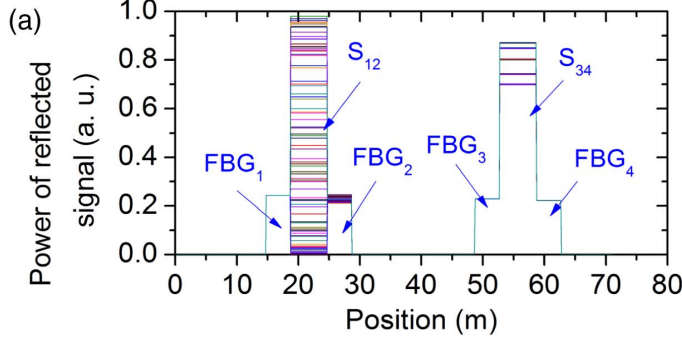

(b)

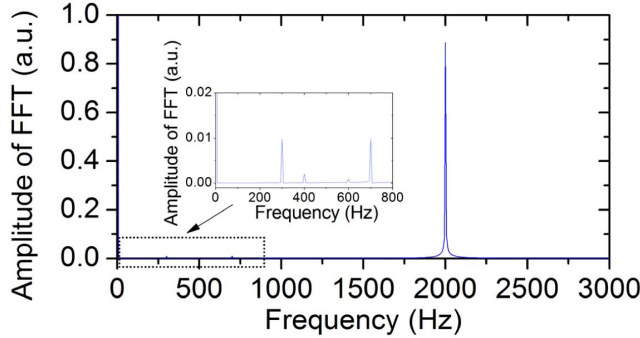

(c)

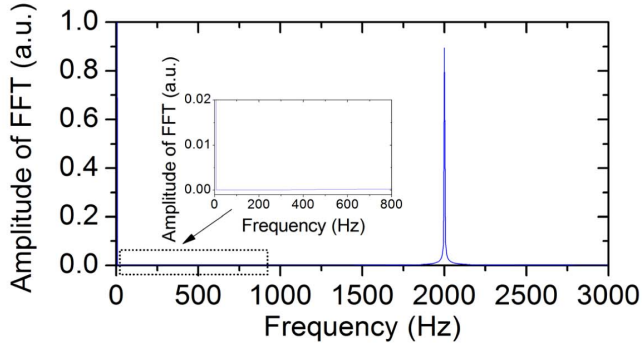

Fig. 3. Simulated (a) reflected signal power versus position over time; (b) FFT for zone $S_{34}$ at $55 \mathrm{~m}$ (inset: zoom 0-800 Hz); and (c) FFT for zone $S_{34}$ at $55 \mathrm{~m}$ after applying the compensation formula (inset: zoom $0-800 \mathrm{~Hz})$ with a $300 \mathrm{~Hz} / 700 \mathrm{~Hz}(2 \mathrm{kHz})$ vibration applied at the first (second) FBG pair.

In zone $S_{34}$, spectral shadowing may appear due to the perturbation applied on $\mathrm{FBG}_{2}$ by the shaker SHR1, resulting in a time-varying transmission coefficient, $\boldsymbol{t}_{\mathbf{2}}$, of $\mathrm{FBG}_{2}$. Figure $3(\mathrm{~b})$ shows the FFT result at $55 \mathrm{~m}$, i.e., at the midpoint of the interference zone $S_{34}$. Three frequencies are clearly distinguished: 2, 300, and $700 \mathrm{~Hz}$, the last two with lower amplitudes. Additionally, the frequencies 400 and $600 \mathrm{~Hz}$ can be noted. As the FBGs present overlapping spectral properties, the interference signal generated by the second pair contains the spectral information of the previous FBG. For this reason, the 300, 700,400 (difference frequency), and $600 \mathrm{~Hz}$ (second harmonic of $300 \mathrm{~Hz}$ ) components are noticed in the FFT, although no vibration at these frequencies are applied at that position.

To avoid this undesirable effect, Eq. (7) is applied to eliminate the influence of spectral shadowing, using the simulated detected power $P_{A B}, P_{A}$, and $P_{B}$ over time at the midpoints of the three zones $\left(S_{34}, \mathrm{FBG}_{3}\right.$, and $\left.\mathrm{FBG}_{4}\right)$ near $55 \mathrm{~m}$. In Fig. 3(c), the FFT of the signal obtained using. Eq. (7) for the second pair is presented. The signal acquired after applying the compensation formula is dimensionless, and power variations are cancelled out. Spectral information about the local perturbation is not removed because it is contained in the phase term $\Delta \varphi(t)+\theta(t)$. As shown in Fig. 3(c), the 300 and $700 \mathrm{~Hz}$ components, and their mixing products and harmonics, are suppressed.

The above analysis procedure has also been applied on experimental phase-OTDR traces recorded under similar conditions. 


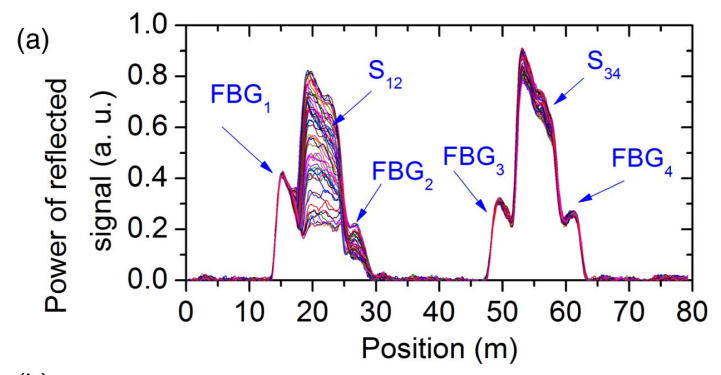

(b)

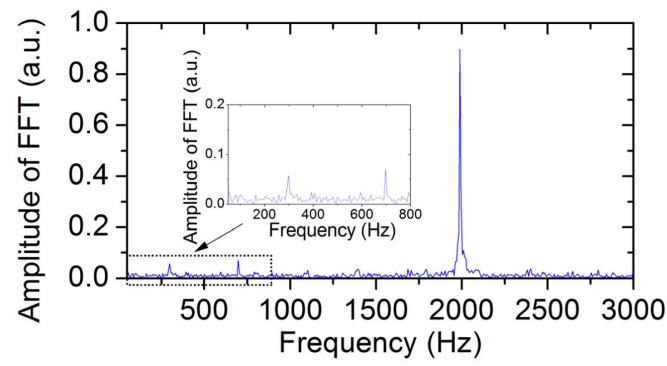

(c)

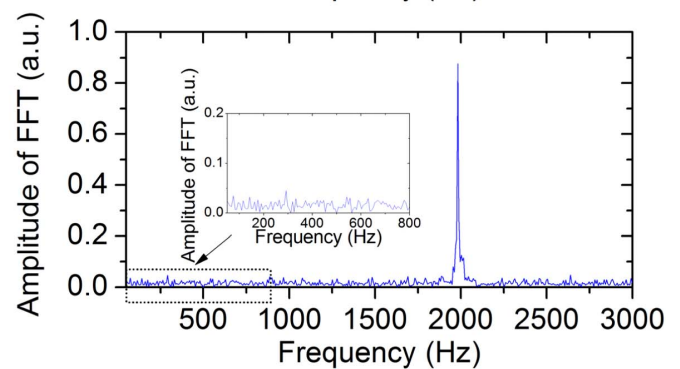

Fig. 4. Detected (a) reflected signal power versus position over time; (b) FFT for zone $S_{34}$ at $55 \mathrm{~m}$ (inset: zoom 0-800 Hz); and (c) FFT for zone $S_{34}$ at $55 \mathrm{~m}$ after applying the compensation formula (inset: zoom $0-800 \mathrm{~Hz})$ with a $300 \mathrm{~Hz} / 700 \mathrm{~Hz}(2 \mathrm{kHz})$ vibration applied at the first (second) FBG pair.

The experimental traces and the FFT obtained for the $S_{34}$ zones are presented in Figs. 4(a) and 4(b), respectively. The 300 and $700 \mathrm{~Hz}$ components are detected within the $S_{34}$ zone. As in the simulation analysis, the compensation using Eq. (7) has been applied to the detected power over time $P_{A B}, P_{A}$, and $P_{B}$ at the midpoints of the three zones $S_{34}, \mathrm{FBG}_{3}$, and $\mathrm{FBG}_{4}$, demonstrating its capability to suppress the 300 and $700 \mathrm{~Hz}$ components as shown in Fig. 4(c). The suppression of the spectral shadowing effect is verified.

Considering the reflection and transmission coefficients of the FBGs, the loss of the connecting fibers and the available dynamic range of the phase-OTDR, the maximum number of FBG pairs can be estimated. Assuming identical FBGs, a fiber loss of $0.2 \mathrm{~dB} / \mathrm{km}$, a maximum detectable power of $100 \mu \mathrm{W}$, and taking the experimental RMS noise level of $0.50 \mu \mathrm{W}$ into account, calculations have been performed for FBG reflectivities of $1 \%, 0.1 \%$, and $0.01 \%$. Provided that each FBG reflects the same power, $P$, the interference peak for a pair of identical FBGs will be a maximum 4P as given by Eq. (6). The results of the calculations are shown in Fig. 5, where the detected reflected power versus FBG pair number is displayed for both maximum interference and single FBG peaks. The input power was set so that the full dynamic range of the detector is utilized, and a minimum detection limit (green horizontal line) was set to $5 \mathrm{~dB}$ above the experimental RMS noise level.

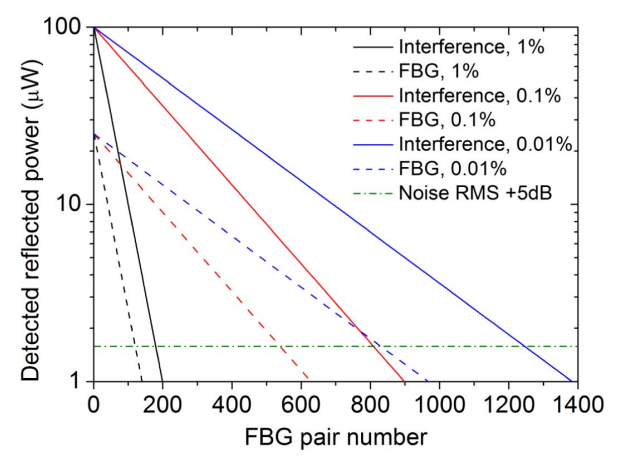

Fig. 5. Simulated detected reflected power versus FBG pair number.

With respect to the power detected from single FBGs, it is concluded that a maximum $530 \mathrm{FBG}$ pairs can theoretically be interrogated with a $0.1 \%$ reflectivity setup (red dashed line). If the reflectivity is decreased to $0.01 \%$ (blue dashed line), the possible number of FBG pairs increases to 830 .

In conclusion, this letter presents a technique to suppress the spectral shadowing effect when interrogating an array of weakly reflective FBGs pairs by a phase-OTDR. For each FBG pair, the proposed method involves the readout of three power values of the phase-OTDR trace: the detected reflected power from each of the two FBGs and the power corresponding to their interference signal. The method has been validated by means of simulations. It has also been experimentally verified by applying it to a proof-of concept phase-OTDR system in the context of vibration measurements. The obtained successful results confirm the effectiveness of the proposed method. A subsequent analysis estimates that the number of FBG pairs that can be interrogated using this technique is nearly 800 with identical FBGs of $0.02 \%$ reflectivity.

Funding. BEWARE Fellowships/Academia (1510633); AEI/FEDER Spanish Government (TEC 2016-76021-C2-1-R).

\section{REFERENCES}

1. J. M. López-Higuera, L. R. Cobo, A. Q. Incera, and A. Cobo, J. Lightwave Technol. 29, 587 (2011).

2. H. N. Li, D. S. Li, and G. B. Song, Eng. Struct. 26, 1647 (2004).

3. R. C. Tennyson, A. A. Mufti, S. Rizkalla, G. Tadros, and B. Benmokrane, Smart Mater. Struct. 10, 560 (2001).

4. M. Majumder, T. K. Gangopadhyay, A. K. Chakraborty, K. Dasgupta, and D. K. Bhattacharya, Sens. Actuators A 147, 150 (2008).

5. X. Bao and C. Liang, Sensors 12, 8601 (2012).

6. J. C. Juarez, E. W. Maier, K. N. Choi, and H. F. Taylor, J. Lightwave Technol. 23, 2081 (2005).

7. F. Peng, N. Duan, Y. J. Rao, and J. Li, IEEE Photon. Technol. Lett. 26 2055 (2014).

8. T. Liu, F. Wang, Q. Yuan, Y. Liu, L. Zhang, and X. Zhang, in 16th International Conference on Optical Communications and Networks (ICOCN) (IEEE, 2017), pp. 1.

9. C. Wang, Y. Shang, X.-H. Liu, C. Wang, H.-H. Yu, D.-S. Jiang, and G.-D. Peng, Opt. Express 23, 29038 (2015).

10. F. Zhu, Y. Zhang, L. Xia, X. Wu, and X. Zhang, J. Lightwave Technol. 33, 4775 (2015).

11. K. Yuksel, V. Moeyaert, P. Mégret, and M. Wuilpart, IEEE Sens. J. 12, 988 (2012).

12. T. Erdogan, J. Lightwave Technol. 15, 1277 (1997). 